\title{
New exoplanets from the SuperWASP-North survey
}

\author{
F. Faedi ${ }^{1}$, S. C. C. Barros ${ }^{1}$, D. Pollacco ${ }^{1}$, E. K. Simpson ${ }^{1}$, J. McCormac ${ }^{1}$, \\ V. Moulds ${ }^{1}$, C. Watson ${ }^{1}$, I. Todd ${ }^{1}$, F. Keenan ${ }^{1}$, A. Fitzsimmons ${ }^{1}, \&$ the \\ WASP Consortium \\ ${ }^{1}$ Astrophysics Research Centre, School of Mathematics 83 Physics, Queen's
University Belfast, University Road, Belfast, BT7 1NN, UK [f.faedi@qub.ac.uk]
}

Abstract. We present the current status of the WASP search for transiting exoplanets, focusing on recent planet discoveries from SuperWASP-North and the joint equatorial region $(-20 \leq$ Dec $\leq+20)$ observed by both WASP telescopes. We report the results of monitoring of WASP planets, and discuss how these contribute to our understanding of planet properties and their diversity.

\section{Introduction}

The importance of transiting extra-solar planets is related to their geometrical configuration which allows us to derive a wealth of information. The transit geometry severely constrains the orbital inclination of the planets, which in turn allows to derive their masses (when combined to radial velocity measurements), their radii and thus their average densities. Even just an estimation of planet bulk physical properties gives insight into their composition (Guillot 2005; Fortney et al. 2007) and can be used to put observational constraints on theoretical models of planetary structure and formation. In addition, transiting systems offer a potential for measuring planetary emission spectra via observations of secondary eclipses (e.g. Charbonneau et al. 2008), while transit spectroscopy gives us an insight into the composition of exoplanets atmospheres (Charbonneau et al. 2002; Vidal-Madjar et al. 2003; Swain et al. 2009).

The more than 100 transiting planets discovered to date show a remarkable variety in their physical and dynamical characteristics, such as the extreme eccentricity of HD 80606b (Naef et al. 2001; Laughlin et al. 2009; Moutou et al. 2009; Fossey et al. 2009; Garcia-Melendo \& McCullough 2009), the ultra-short period of WASP-19b (Hebb et al. 2010) and the puzzlingly low densities of WASP-17b (Anderson et al. 2010) and Kepler-7b (Latham et al. 2010). The range of parameters which can be explored with transiting systems is restricted by observational and instrumental limitations. However, many challenges faced by the current surveys are being overcome by the ability to decrease systematic noise and optimise follow-up strategies. Indeed, in the case of SuperWASP-North the reduction of systematic noise yielded an increase in planet detection.

In this proceedings we present some of the latest discoveries of exoplanets by the SuperWASP-Noth survey and the joint equatorial strip. These newly discovered transiting planets are important to constrain theoretical models of planetary formation and

This is an Open Access article distributed under the terms of the Creative Commons Attribution-Noncommercial License 3.0, which permits unrestricted use, distribution, and reproduction in any noncommercial medium, provided the original work is properly cited. 
dynamical evolution, since they belong to a not, as yet, well sampled region of the parameter space.

\section{SuperWASP-North survey}

The SuperWASP-North observatory in La Palma consists of 8 cameras each with a Canon 200-mm f/1.8 lens coupled to an Andor e2v 2048×2048 pixel back-illuminated CCD (Pollacco et al. 2006). This configuration gives a pixel scale of $13.7^{\prime \prime} /$ pixel which corresponds to a field of view of $7.8 \times 7.8$ square degrees per camera.

In October 2008, we introduced an electronic focus control and we also started stabilisation of the temperature of the SuperWASP-North camera lenses. Prior to this upgrade, night-time temperature variations affected the focal length of the WASP lenses, altering the FWHM of stars. This introduced trends in the data which mimic partial transits events, especially at the beginning and end of the night when the temperature variation is more extreme. These effects are not corrected by our detrending algorithms SYSREM (Tamuz, Mazeh \& Zucker 2005, Cameron et al. 2006) and TFA (Kovács, Bakos, \& Noyes 2005) because they are position-dependent and affect groups of stars differently. In order to reduce this source of systematic noise, heating strips were placed around each lens so that their temperature is maintained above ambient at 21 degrees. Besides the stabilisation of the temperature we also significantly improved the focus of each of the lenses, which can now be remotely controlled. This upgrade has been crucial to improve the signal-to-noise of WASP light-curves, allowing the detection of planets, previously hidden in the RMS scatter of the data (e.g. WASP-38b, Barros et al. 2010).

Moreover, since January 2008 both instruments, WASP-South and SuperWASP-North, have been monitoring an equatorial region of sky $(-20 \leq \mathrm{Dec} \leq+20)$ significantly increasing the amount of data collected on each planet candidate. This has been a key element, together with our improvements to the SuperWASP-North system, for the detection of the most recent WASP planets. The WASP planets detection rate is better than 1 planet for $6-7$ candidates.

\subsection{Follow-up campaign}

We perform our spectroscopic follow-up campaign using the highly pressure-stabilised Echelle spectrographs SOPHIE mounted on the $1.93 \mathrm{~m}$ telescope at the Observatoire de Haute Provence (Perruchot et al. 2008; Bouchy et al. 2009), and CORALIE on the 1.2m Swiss Euler telescope at ESO La Silla Observatory in Chile (Baranne et al. 1996; Queloz et al. 2000; Pepe et al. 2002). In addition, we also use the Fiber-Fed Echelle spectrograph (FIES) mounted on the $2.56 \mathrm{~m}$ Nordic Optical Telescope in La Palma.

We obtain high precision high signal-to-noise transit light-curves using the LCOGT 2.0m Faulkes-North and South telescopes situated on Haleakala, Hawaii, and Siding Springs, Australia, respectively; the robotic 2.0-m Liverpool Telescope (LT) equipped with the RISE frame transfer CCD, located on La Palma (Gibson et al. 2008; Steele et al. 2008), and finally the Euler-Swiss telescope in La Silla.

\section{New SuperWASP discoveries}

Here we present an overview of the newly discovered WASP planets. 
Detection and Dynamics of Transiting Exoplanets
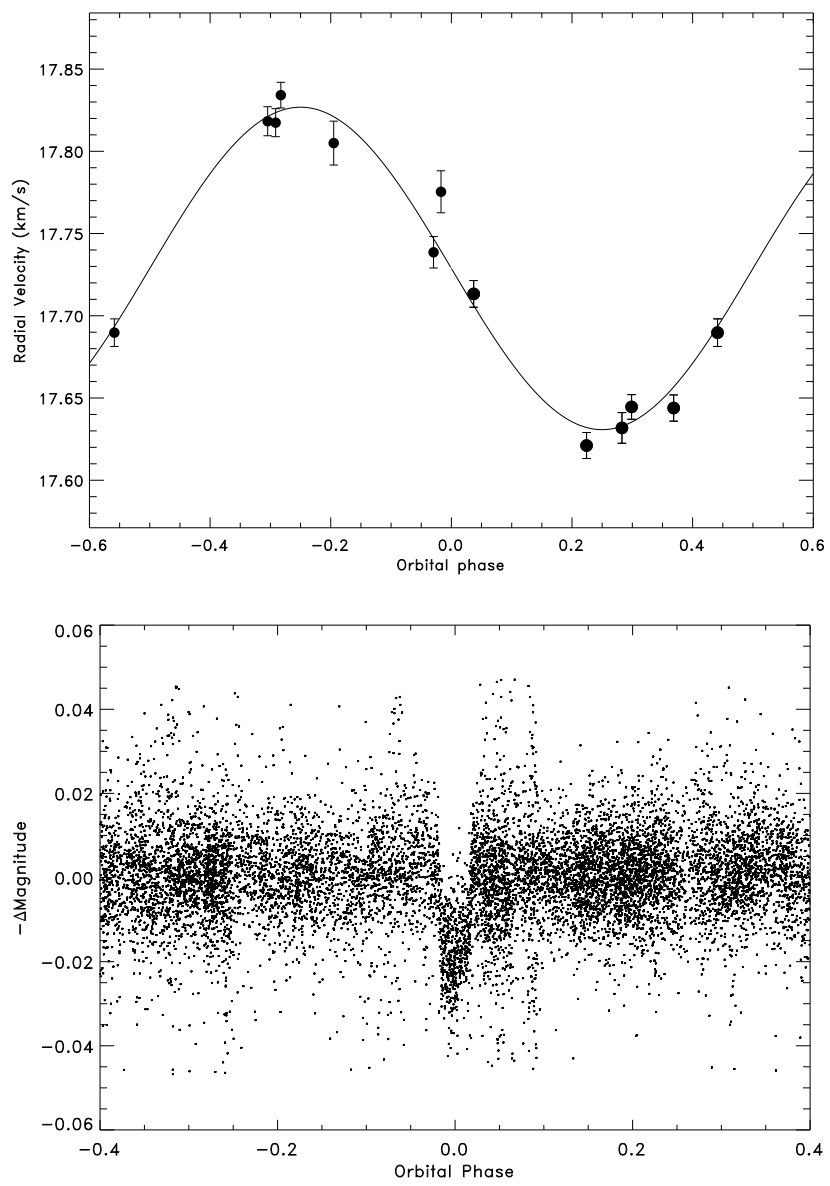

Figure 1.: Upper panel: WASP-35b radial velocity curve FIES and CORALIE data. Lower panel: WASP discovery light-curve. Follow-up photometry is scheduled for WASP-35b.

\subsection{WASP-37b}

WASP-37b (Simpson et al. 2010) is a hot Jupiter planet with a radius of $\mathrm{R}_{p l}=1.16_{-0.06}^{+0.07} \mathrm{R}_{J u p}$, a mass of $\mathrm{M}_{p l}=1.80 \pm 0.17 M_{J u p}$ and an orbital period of $\mathrm{P}=3.6$ days. It has a high surface gravity compared to other planets with similar orbital periods $\left(\log g_{p l}=3.48_{-0.04}^{+0.03}\right.$ ), which lies significantly above the period-gravity correlation proposed by Southworth (2010). WASP-37b is orbiting a very old (age 11 Gyr) metal poor $([\mathrm{Fe} / \mathrm{H}]=-0.4 \pm 0.12 \mathrm{dex})$ star , firmly in the tail of the metallicity distribution for exoplanet host stars, which is probably a member of the thick disc population. Even if theoretical models of Fortney et al. (2007) and Baraffe et al. (2008) do not cover the age range of the system, because planetary radii are thought to decrease with age, we find that WASP-37b has an inflated radius and probably no core. This is consistent with the correlation between core mass and metallicity (Guillot et al. 2006; Burrows et al. 2007) given the low stellar metallicity. 

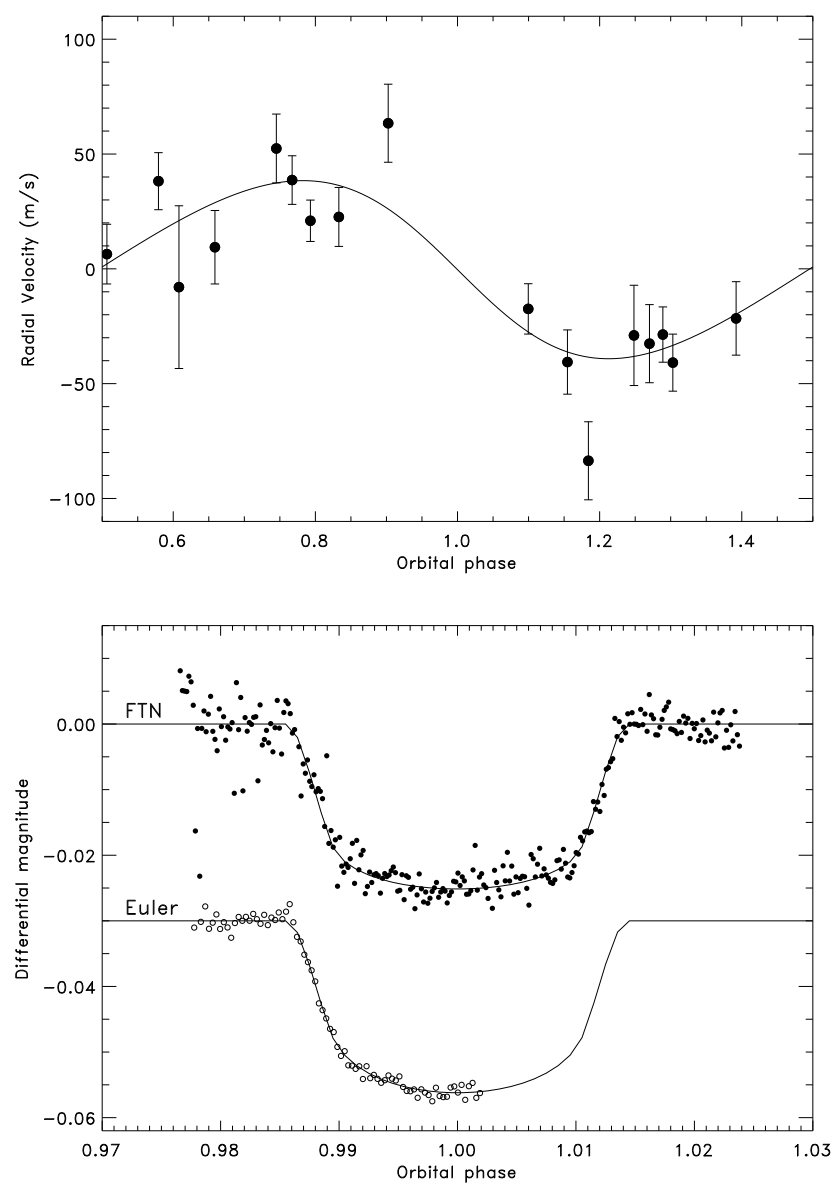

Figure 2.: Upper panel: WASP-39b radial velocity curve SOPHIE and CORALIE data. Lower panel: FTN and Euler light-curves.

More details on the system, follow-up spectroscopy and photometry are presented in Simpson et al. (2010).

\subsection{WASP-38b}

WASP-38b (Barros et al. 2010) is a long period $(\mathrm{P}=6.87 \mathrm{~d})$, massive $\left(2.69 \pm 0.06 \mathrm{M}_{J u p}\right)$ planet in an eccentric orbit $(e=0.031)$. WASP-38b does not suffer from the radius anomaly mentioned above $\left(\mathrm{R}_{p l}=1.09 \pm 0.03 \mathrm{R}_{J u p}\right)$ and according to Fortney et al. (2008) belongs to the 'pL' class of planets, which show no temperature inversion in their atmosphere. WASP-38b is the forth exoplanet with $\mathrm{P}>6$ days discovered in a ground-based transit survey (the remaining three are WASP-8b, Queloz et al. 2010, HAT-P-15b Kovacs et al. 2010, and HAT-P-17b Howard et al. 2010). The smaller transit probability, the longer duty cycle and transit duration of these systems, coupled with the restricted observing time from a single site make long period systems more challenging to detect. To increase the duty cycle of the observations, telescope networks spread in geographic longitude or space-based surveys are needed. In the case of WASP- 

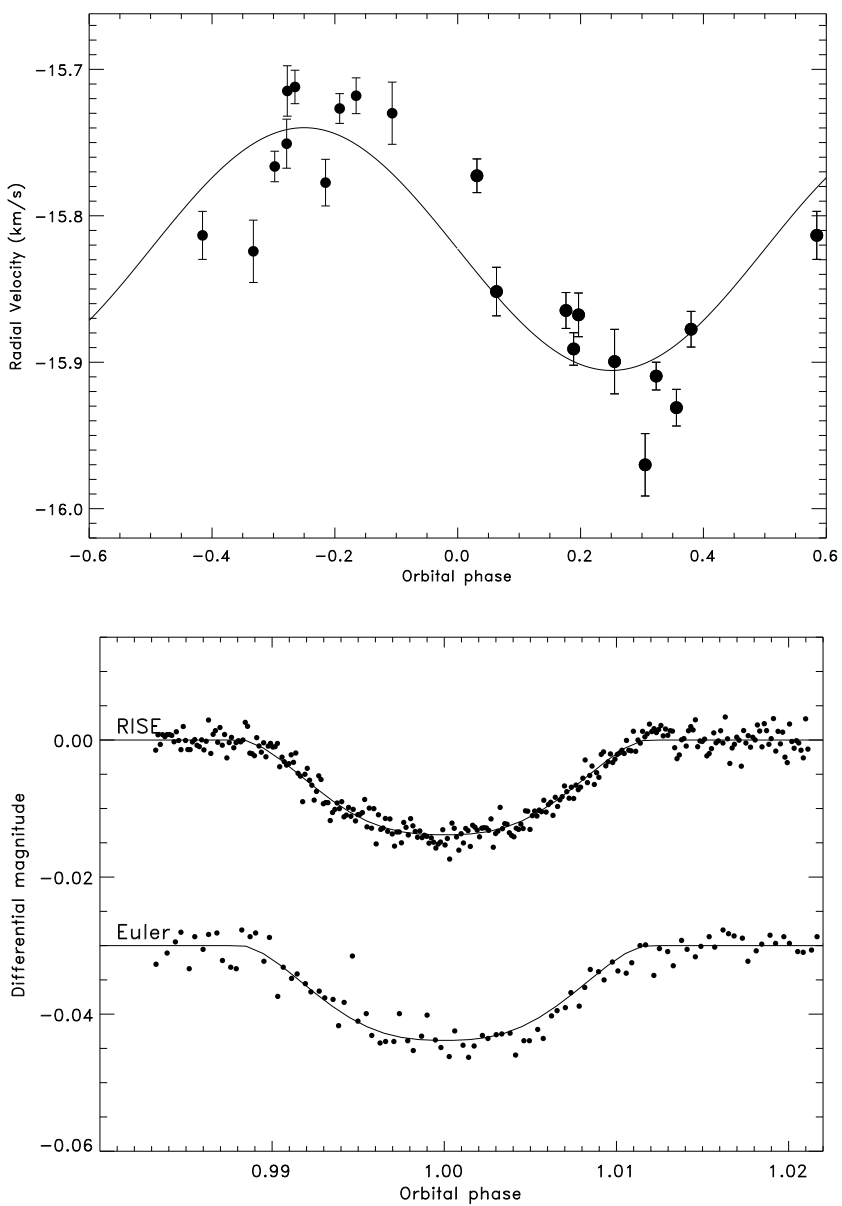

Figure 3.: Upper panel: WASP-40b radial velocity curve SOPHIE and CORALIE data. Lower panel: follow-up photometry with RISE and Euler.

$38 \mathrm{~b}$, it was very important to reduce the systematic noise which ultimately allowed the discovery of the planet. However, the selection effects might be hiding a real decrease of the number of planets at longer periods. In fact, from radial velocity surveys there appears to be a depletion of planets between 0.1-1AU (Udry et al. 2003).

Details of the system, as well as, follow-up spectroscopy, and photometry are presented in Barros et al. (2010).

\subsection{WASP-35b, WASP-39b and WASP-40b}

WASP-35b, WASP-39b and WASP40b are all sub-Jupiter mass planets found in the joint equatorial region of sky observed simultaneously by both WASP telescopes.

Figure 1 shows the radial velocity follow-up curve and the WASP discovery photometry of WASP-35b. This is the first WASP planet identified in the joint equatorial region and it is a sub-Jupiter mass planet with an estimated mass of $\sim 0.7 \mathrm{M}_{J u p}$ and radius $\sim 1.3 \mathrm{R}_{\text {Jup }}$ orbiting a slightly metal poor star every 3 days (Enoch et al. in prep.). 
We present our discovery of WASP-39b in Figure 2. This is a Saturn mass planet, orbiting a late $\mathrm{G}$ type star every $\sim 4$ days. From the data presented above we obtained a preliminary estimate of the planetary mass of $\sim 0.3 \mathrm{M}_{J u p}$ and radius of $\sim 1.3 \mathrm{R}_{J u p}$. This suggest that WASP-39b is a very low density planet with an inflated radius (Faedi et al. in prep).

Finally, in Figure 3 we present the radial velocity and photometry follow-up data of WASP-40b. WASP-40b is orbiting a late G/early $\mathrm{K}$ dwarf star with an orbital period of $\sim 3$ days, and has an estimated mass of $\sim 0.6 \mathrm{M}_{\text {Jup }}$ and radius of $\sim 1 \mathrm{R}_{\text {Jup }}$. The radial velocity residuals of WASP-40b, after subtracting the fitted model, also suggest the presence of an additional signal. However more data are needed before any conclusion can be drawn (West et al. in prep).

\section{Conclusion}

We have discussed the improvement brought to the SuperWASP-North system, and our observing strategy, which allowed the detection of the transiting extra-solar planets presented in this proceedings. These newly discovered planets are extremely important to the understanding of planetary formation, and evolution, and will help to constrain theoretical models. For example, WASP-39b is a Saturn-mass planet orbiting a late $\mathrm{G}$ star with a highly inflated radius, more than $20 \%$ larger than the $\mathrm{R}_{p l}$ obtained by comparison with the Fortney et al. (2007) model for a coreless planet of a similar mass and orbital distance. WASP-40b instead, is a slightly more massive planet, orbiting a late G/Early $\mathrm{K}$ star, and does not appear to show the radius anomaly. In addition, WASP-38b is one of the longest period transiting planet discovered from ground and WASP-37b's host stars is one of the older stars, of very low metallicity, hosting a transiting planet suggesting that giant planet formation was taking place when the Milky Way was still relatively young.

To date there are 150-200 planet candidates in the WASP archive which will be followed up in the forecoming seasons. We therefore expect, more interesting systems to be dicovered by SuperWASP in the near future.

\section{References}

Anderson, D. R., et al. 2010, ApJ, 709, 159

Baraffe, I., Chabrier, G., \& Barman, T. 2008, A\&A, 482, 315

Baranne, A., et al. 1996, A\&AS, 119, 373

Barros, S. C. C., et al. 2010, arXiv:1010.0849

Bouchy, F., et al. 2009, A\&A, 505, 853

Burrows, A., Hubeny, I., Budaj, J., \& Hubbard, W. B. 2007, ApJ, 661, 502

Charbonneau, D., Brown, T. M., Noyes, R. W., \& Gilliland, R. L. 2002, ApJ, 568, 377

Charbonneau, D., Knutson, H. A., Barman, T., Allen, L. E., Mayor, M., Megeath, S. T., Queloz, D., \& Udry, S. 2008, ApJ, 686, 1341

Collier Cameron, A., et al. 2006, MNRAS, 373, 799

Fortney, J. J., Marley, M. S., \& Barnes, J. W. 2007, ApJ, 659, 1661

Fossey, S. J., Waldmann, I. P., \& Kipping, D. M. 2009, MNRAS, 396, L16

Hebb, L., et al. 2010, ApJ, 708, 224

Howard, A. W., et al. 2010, arXiv:1008.3898 
Kovács, G., Bakos, G., \& Noyes, R. W. 2005, MNRAS, 356, 557

Kovács, G., et al. 2010, ApJ, 724, 866

Garcia-Melendo, E., \& McCullough, P. R. 2009, ApJ, 698, 558

Gibson, N. P., et al. 2008, A\&A, 492, 603

Guillot, T. 2005, Annual Review of Earth and Planetary Sciences, 33, 493

Guillot, T., Santos, N. C., Pont, F., Iro, N., Melo, C., \& Ribas, I. 2006, A\&A, 453, L21

Latham, D. W., et al. 2010, ApJ, 713, L140

Laughlin, G. 2009, Nature , 459, 781

Moutou, C., et al. 2009, A\&A, 498, L5

Naef, D., et al. 2001, A\&A, 375, L27

Pepe, F., Mayor, M., Galland, F., Naef, D., Queloz, D., Santos, N. C., Udry, S., \& Burnet, M. 2002, A\&A, 388, 632

Perruchot, S., et al. 2008, Proceedings of the SPIE, 7014

Pollacco, D., et al. 2006, Ap\&SS, 304, 253

Queloz, D., et al. 2000, A\&A, 354, 99

Queloz, D., et al. 2010, A\&A, 517, L1

Simpson, E. K., et al. 2010, arXiv:1008.3096

Southworth, J. 2010, MNRAS, 408, 1689

Steele, I. A., Bates, S. D., Gibson, N., Keenan, F., Meaburn, J., Mottram, C. J., Pollacco, D., \& Todd, I. 2008, Proceedings of the SPIE, 7014,

Swain, M. R., Vasisht, G., Tinetti, G., Bouwman, J., Chen, P., Yung, Y., Deming, D., \& Deroo, P. 2009, ApJ, 690, L114

Tamuz, O., Mazeh, T., \& Zucker, S. 2005, MNRAS, 356, 1466

Udry, S., Mayor, M., \& Santos, N. C. 2003, A\&A, 407, 369

Vidal-Madjar, A., Lecavelier des Etangs, A., Désert, J.-M., Ballester, G. E., Ferlet, R., Hébrard, G., \& Mayor, M. 2003, Nature , 422, 143 